\title{
THE CIRCUMGALACTIC MEDIUM OF SUBMILLIMETER GALAXIES. I. FIRST RESULTS FROM A RADIO-IDENTIFIED SAMPLE
}

\author{
Hai Fu $^{1}$, J. F. Hennawi ${ }^{2}$, J. X. Prochaska ${ }^{3}$, R. Mutel ${ }^{1}$, C. Casey ${ }^{4}$, A. Cooray ${ }^{5}$, D. Kereš ${ }^{6}$, Z.-Y. Zhang ${ }^{7,8}$, D. Clements ${ }^{9}$, \\ J. Isbell $^{1}$, C. Lang $^{1}$, D. McGinnis ${ }^{1}$, M. J. Michałowski ${ }^{7}$, K. Mooley ${ }^{10}$, D. Perley ${ }^{11}$, A. Stockton ${ }^{12}$, And D. Thompson ${ }^{13}$ \\ ${ }^{1}$ Department of Physics \& Astronomy, University of Iowa, Iowa City, IA 52242, USA \\ ${ }^{2}$ Max-Planck-Institut fur Astronomie, Heidelberg, Germany \\ ${ }^{3}$ Department of Astronomy and Astrophysics, UCO/Lick Observatory, University of California, 1156 High Street, Santa Cruz, CA 95064, USA \\ ${ }^{4}$ Department of Astronomy, the University of Texas at Austin, 2515 Speedway Blvd, Stop C1400, Austin, TX 78712, USA \\ 5 Department of Physics and Astronomy, University of California, Irvine, CA 92697, USA \\ ${ }^{6}$ Department of Physics, Center for Astrophysics and Space Sciences, University of California at San Diego, 9500 Gilman Drive, La Jolla, CA 92093, USA \\ Institute for Astronomy, University of Edinburgh, Royal Observatory, Blackford Hill, Edinburgh EH9 3HJ, UK \\ ${ }^{8}$ ESO, Karl Schwarzschild Strasse 2, D-85748 Garching, Munich, Germany \\ ${ }^{9}$ Astrophysics Group, Imperial College London, Blackett Laboratory, Prince Consort Road, London SW7 2AZ, UK \\ ${ }^{10}$ Oxford Centre For Astrophysical Surveys, Denys Wilkinson Building, Keble Road, Oxford OX1 3RH, USA \\ ${ }^{11}$ Dark Cosmology Centre, Niels Bohr Institute, University of Copenhagen, Juliane Maries Vej 30, DK-2100 København $\emptyset$, Denmark \\ ${ }^{2}$ Institute for Astronomy, University of Hawaii, 2680 Woodlawn Drive, Honolulu, HI 96822, USA \\ ${ }^{13}$ Large Binocular Telescope Observatory, University of Arizona, 933 N. Cherry Ave, Tucson, AZ 85721, USA \\ Received 2016 June 23; revised 2016 August 8; accepted 2016 August 19; published 2016 November 15
}

\begin{abstract}
We present the first results from an ongoing survey to characterize the circumgalactic medium (CGM) of massive high-redshift galaxies detected as submillimeter galaxies (SMGs). We constructed a parent sample of 163 SMGQSO pairs with separations less than $\sim 36^{\prime \prime}$ by cross-matching far-infrared-selected galaxies from Herschel with spectroscopically confirmed QSOs. The Herschel sources were selected to match the properties of the SMGs. We determined the sub-arcsecond positions of six Herschel sources with the Very Large Array and obtained secure redshift identification for three of those with near-infrared spectroscopy. The QSO sightlines probe transverse proper distances of 112, 157, and $198 \mathrm{kpc}$ at foreground redshifts of 2.043, 2.515, and 2.184, respectively, which are comparable to the virial radius of the $\sim 10^{13} M_{\odot}$ halos expected to host SMGs. High-quality absorption-line spectroscopy of the QSOs reveals systematically strong H I Ly $\alpha$ absorption around all three SMGs, with rest-frame equivalent widths of $\sim 2-3 \AA$. However, none of the three absorbers exhibit compelling evidence for optically thick $\mathrm{H}$ I gas or metal absorption, in contrast to the dominance of strong neutral absorbers in the CGM of luminous $z \sim 2$ QSOs. The low covering factor of optically thick H I gas around SMGs tentatively indicates that SMGs may not have as prominent cool gas reservoirs in their halos as the coeval QSOs and that they may inhabit less massive halos than previously thought.
\end{abstract}

Key words: galaxies: halos - intergalactic medium - quasars: absorption lines

\section{INTRODUCTION}

The first millijansky-level submillimeter surveys discovered a population of distant submillimeter-bright galaxies (SMGs), namely, unresolved sources with $850 \mu \mathrm{m}$ flux density $\left(S_{850}\right)$ greater than 3-5 mJy (Smail et al. 1997; Barger et al. 1998; Hughes et al. 1998; Eales et al. 1999). The SMGs selected at wavelengths between $850 \mu \mathrm{m}$ and $1 \mathrm{~mm}$ are intense starbursts (SFR $\gtrsim 500 M_{\odot} \mathrm{yr}^{-1}$ ) at a median redshift of $z \sim 2.5$ (Chapman et al. 2005; Wardlow et al. 2011; Smolčić et al. 2012; Yun et al. 2012). The intense star formation is dust-enshrouded, so that the SMGs radiate most of their bolometric luminosity in the far-infrared (IR). The observed molecular and stellar emission indicates that they are massive gas-rich galaxies $\left(M_{\mathrm{gas}} \sim M_{\mathrm{star}} \sim 10^{11} M_{\odot}\right.$; e.g., Michalowski et al. 2010; Hainline et al. 2011; Bothwell et al. 2013), but the typical halo mass of SMGs remains uncertain, with estimates ranging from $10^{12}$ to $10^{13} M_{\odot}$. Two lines of evidence suggest that SMGs may inhabit dark matter halos as massive as $\sim 10^{13} M_{\odot}$ : (1) their strong clustering strength estimated from either the angular two-point correlation function (e.g., Scott et al. 2006; Weiß et al. 2009) or the cross-correlation function between SMGs and other high-redshift galaxies (e.g., Hickox et al. 2012), and (2) their high stellar mass and the $M_{\text {star }}-M_{\text {halo }}$ relation from abundance matching $\left(M_{\text {halo }}=6 \times 10^{12} M_{\odot}\right.$ for $M_{\text {star }}=10^{11} M_{\odot}$ at $z=2$; e.g., Behroozi et al. 2010). However, because source blending due to the large beams of single-dish (sub)millimeter telescopes may have significantly elevated clustering strength (Cowley et al. 2016) and the stellar mass estimates remain uncertain within an order of magnitude (e.g., Hainline et al. 2011; Michałowski et al. 2012; Targett et al. 2012), it is possible that a typical SMG may inhabit much less massive halos $\left(\sim 10^{12} M_{\odot}\right)$.

SMGs are absent in the local universe, and it is commonly thought that they have evolved into massive ellipticals today (e.g., Blain et al. 2004; Toft et al. 2014). To understand the evolution of SMGs, it is imperative to know how long the observed intense star formation would last. For $10^{13} M_{\odot}$ halos at $z=2.5$, the average baryonic accretion rate from the mass growth rate of dark matter halos is $\dot{M}_{\text {gas }} \equiv 0.18 \times$ $\dot{M}_{\text {halo }} \simeq 1.4 \times 10^{3} M_{\odot} \mathrm{yr}^{-1}$ (Neistein \& Dekel 2008; Bouché et al. 2010). In such massive halos, it is expected that most of the baryons will be shock-heated to the virial temperature of the halo $\left(\sim 10^{7} \mathrm{~K}\right)$, so that only a small fraction of the accreted gas can actually cool and accrete onto galaxies (e.g., Keres et al. 2005; Dekel \& Birnboim 2006). Therefore, the ongoing gas accretion is unlikely to sustain the extreme SFRs. Without a comparable gas supply rate, the SFR would decline with an $e$-folding timescale of only $200 \mathrm{Myr}$ 
$\left(2 M_{\text {gas }}\right.$ SFR; e.g., Greve et al. 2005; Tacconi et al. 2008; Ivison et al. 2011; Bothwell et al. 2013; Fu et al. 2013). At such a rate, the SMGs would become red sequence galaxies in only a Gyr or $5 e$-folding times. ${ }^{14}$ Such a short transitional time of a significant high-redshift star-forming population might help explain the rapid build-up of the massive end of the red sequence at $z>1$ (e.g., Ilbert et al. 2013). Starbursts thus provide an alternative mechanism to the QSO-mode feedback (e.g., Silk \& Rees 1998; Di Matteo et al. 2005) to form red and dead galaxies. Note that both mechanisms still require feedback from radio jets (i.e., the maintenance mode; e.g., Fabian 2012; Heckman \& Best 2014) to prevent the hot gaseous halo from cooling. On the other hand, if SMGs were in $10^{12} M_{\odot}$ halos, the intense star formation would also be unsustainable because the gas accretion rate would be only $\sim 110 M_{\odot} \mathrm{yr}^{-1}$ at $z=2.5$.

However, could there be enough cool gas in the circumgalactic medium (CGM) around SMGs to fuel a prolonged starburst phase (e.g., see the simulation of Narayanan et al. 2015)? The CGM of coeval QSOs may give us a hint, because they inhabit comparably massive $\left(\sim 10^{12.6} M_{\odot}\right)$ halos (White et al. 2012). Contrary to the expected dominance of virialized X-ray plasma, absorption line spectroscopy of a statistical sample of $z \sim 2$ projected QSO pairs reveals the prevalence of cool $\left(T \sim 10^{4} \mathrm{~K}\right)$, metal-enriched $\left(Z \geqslant 0.1 Z_{\odot}\right)$, and optically thick Ly $\alpha$ absorbers $\left(N_{\mathrm{H}} \geqslant 10^{17.2} \mathrm{~cm}^{-2}\right)$ extending to at least the expected virial radius of $160 \mathrm{kpc}$ (the "QSO Probing QSO" (QPQ) project: Hennawi et al. 2006a; Hennawi \& Prochaska 2007; Prochaska et al. 2013a, 2013b). The high observed covering factor of the cool CGM gas $(\gtrsim 60 \%)$ in $\sim 10^{12.6} M_{\odot}$ halos has been compared to predictions from numerical simulations. While several studies found that they cannot reproduce the high covering factor around QSOs (Fumagalli et al. 2014; Faucher-Giguère et al. 2015; but see Rahmati et al. 2015), it has been argued that efficient star formation-driven winds from accreted satellite galaxies that interact with cosmological filaments are required to increase the H I covering factor to the observed level, which is resolved only in the highest-resolution cosmological zoom simulations (Faucher-Giguere et al. 2016).

These high-resolution simulations predict that the covering factor is roughly independent of SFR, decreases with redshift, and has relatively large halo-halo variations. In the relevant halo mass range, $\sim 3 \times 10^{12}-10^{13} M_{\odot}$, simulations show $\mathrm{H}$ I covering factors of $\sim 30 \%-80 \%$ with little mass dependence at $z \sim 2$ (Faucher-Giguere et al. 2016). Given the estimated halo mass for QSOs and SMGs, one could expect similar covering factors if these are determined primarily by the interplay between gas infall and star formation-driven outflows. To test this, we exploit QSO absorption line spectroscopy to probe the CGM of SMGs. In Section 2, we present the data sets and the method we used to select projected SMG -QSO pairs. We then describe our follow-up observations in Section 3, including those from radio interferometer imaging, near-infrared spectroscopy, and optical spectroscopy. We present our analysis and results in Section 4, including a comparison between the covering factor of optically thick gas around SMGs and that of $z \sim 2$ QSOs. We summarize the results and conclude in Section 5. Throughout we adopt

\footnotetext{
${ }^{14}$ This is the time it would take to decrease the specific SFR (SFR/ $M_{\text {star }}$ ) from $\sim 10^{-9} \mathrm{Gyr}^{-1}$ for the SMGs at the observed epoch to $\sim 10^{-11} \mathrm{Gyr}^{-1}$ for the red sequence at $z \sim 2$ (Brammer et al. 2009).
}

a $\Lambda$ CDM cosmology with $\Omega_{\mathrm{m}}=0.27, \Omega_{\Lambda}=0.73$, and $H_{0}=70 \mathrm{~km} \mathrm{~s}^{-1} \mathrm{Mpc}^{-1}$.

\section{SELECTION OF PROJECTED SMG-QSO PAIRS}

Because both high-redshift QSOs and SMGs have lownumber counts, we needed large samples of both to come up with a sizable sample of projected SMG-QSO pairs with small angular separations. We compiled 464,866 spectroscopically confirmed QSOs from various surveys-primarily, the Sloan Digital Sky Survey (SDSS; Alam et al. 2015), the 2dF QSO Redshift Survey (Croom et al. 2004), the AGN and Galaxy Evolution Survey (Kochanek et al. 2012), and the MMT Hectospec Redshift Survey of $24 \mu \mathrm{m}$ Sources in the Spitzer First Look Survey (Papovich et al. 2006). Since we selected foreground galaxies that are likely to be at $z>2$ (see the next paragraph), we kept only 102,472 QSOs at $z_{\mathrm{QSO}}>2.5$. The average surface density of these background QSOs is $\sim 10 \mathrm{deg}^{-2}$.

To select the foreground SMGs, we combined source catalogs from a number of wide-area extragalactic surveys carried out by the Herschel ${ }^{15}$ Space Observatory (Pilbratt et al. 2010): the Herschel Multi-tiered Extragalactic Survey (HerMES, $95 \mathrm{deg}^{2}$; Oliver et al. 2012; Wang et al. 2014), the Herschel Astrophysical Terahertz Large Area Survey (HATLAS, $600 \mathrm{deg}^{2}$; Eales et al. 2010; Valiante et al. 2016), the Herschel Large Mode Survey (HeLMS, $301 \mathrm{deg}^{2}$; Oliver et al. 2012; Asboth et al. 2016; C.L. Clarke et al. 2016, in preparation; Nayyeri et al. 2016), and the Herschel Stripe 82 Survey (HerS, $79 \mathrm{deg}^{2}$; Viero et al. 2014). All of these surveys used SPIRE (Spectral and Photometric Imaging Receiver; Griffin et al. 2010) to image the sky at 250, 350, and $500 \mu \mathrm{m}$, and the combined xID250 ${ }^{16}$ catalog contains $1,586,047$ sources covering a total of $767 \mathrm{deg}^{2}$ (the HerS and HeLMS fields overlap by $10 \mathrm{deg}^{2}$ ).

However, most Herschel sources are not SMGs; they are, instead, less luminous dusty star-forming galaxies at lower redshifts $(z<2$; Casey et al. 2012, 2014). To select Herschel sources that are likely to be SMGs, we chose only the subsample that satisfies the following criteria: (1) flux density peak at $350 \mu \mathrm{m}\left(S_{250}<S_{350}\right.$ and $S_{500}<S_{350}$; i.e., "350 $\mu \mathrm{m}$ peakers"), (2) $S_{500}>20 \mathrm{mJy}$, and (3) $>3 \sigma$ detections in all three SPIRE bands. Criterion 1 is essentially a photometric redshift selection because emission from dusts at $T=35 \mathrm{~K}$ would peak at $350 \mu \mathrm{m}$ if redshifted to $z \sim 2.5$. This is confirmed by the blind carbon monoxide (CO $J=1-0)$ survey of a subsample of the brightest $350 \mu$ m peakers $\left(S_{350} \geqslant 115 \mathrm{mJy}\right)$, which has shown a strikingly similar redshift distribution as $850 \mu \mathrm{m}$ selected SMGs $\left(z_{\mathrm{CO}}=2.5 \pm 0.8\right.$; Harris et al. 2012). But note that most of these bright sources are strongly lensed and they do not overlap with our sample. Criterion 2 is introduced to ensure that the Rayleigh-Jeans extrapolation would give $S_{850}>3 \mathrm{mJy}$, the classic definition of an SMG, given a typical power-law slope of 3.5 for a modified blackbody with a frequency-dependent absorption cross section $\left(\kappa \propto \nu^{1.5}\right)$. Criterion 3 ensures that all of the sources we considered are statistically significant. This is necessary because the image depth varies substantially from field to

\footnotetext{
${ }^{15}$ Herschel is an ESA space observatory with science instruments provided by European-led Principal Investigator consortia and with important participation from NASA.

16 Fluxes of 250,350 and $500 \mu \mathrm{m}$ were all extracted at source positions detected on the $250 \mu \mathrm{m}$ map (e.g., Roseboom et al. 2010; Rigby et al. 2011).
} 


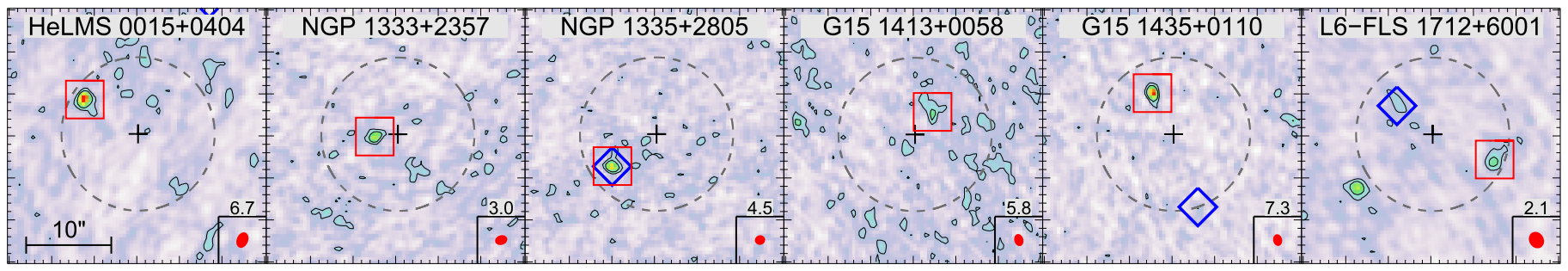

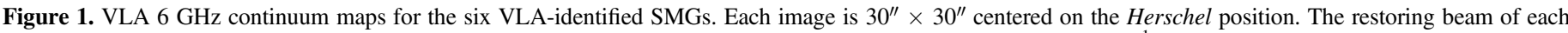

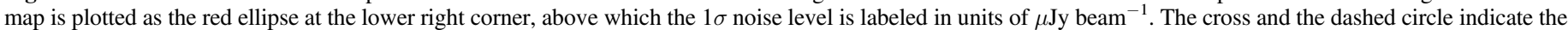

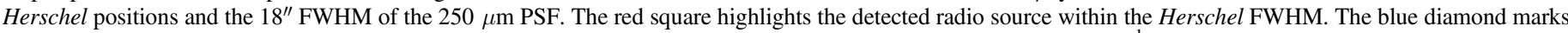

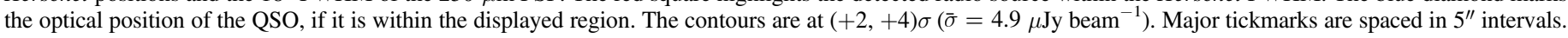
North is up and east is left for all panels.

field, ranging from $\sigma_{500}=15 \mathrm{mJy}$ beam $^{-1}$ for the large HeLMS and HerS fields (confusion noise included; Oliver et al. 2012; Viero et al. 2014) to confusion limited with $\sigma_{500}=6.8$ mJy beam $^{-1}$ for the deeper HerMES fields (Nguyen et al. 2010). Given the range of observed far-IR SEDs at $z=2$ from Casey et al. (2012), our color selection and the high threshold on the $500 \mu \mathrm{m}$ flux density ensure that $\sim 95 \%$ of our sample would be classified as SMGs if they were observed at $870 \mu \mathrm{m}$. Nevertheless, we should keep in mind that the Herschel-selected SMGs are a subsample of SMGs and they likely cover a smaller range of dust temperatures than $870 \mu \mathrm{m}$ selected SMGs (e.g., Hwang et al. 2010; Magnelli et al. 2012). Only 70,823 Herschel sources remained after this selection. The average surface density of $92 \mathrm{deg}^{-2}$ is five times lower than the observed $870 \mu \mathrm{m}$ source count above $S_{870} \gtrsim 3 \mathrm{mJy}$ ( $\sim 500 \mathrm{deg}^{-2}$; Coppin et al. 2006; Weiß et al. 2009). This is not surprising given that almost half of the total Herschel area is only $10 \%-20 \%$ complete at $S_{500}=20 \mathrm{mJy}$. Note that this incompleteness in the Herschel catalogs is not a concern for compiling a sample of SMG-QSO pairs.

We identified 230 projected SMG-QSO pairs with angular separations $\left(\theta_{250}\right)^{17}$ of $5^{\prime \prime} \leqslant \theta_{250} \leqslant 36^{\prime \prime}$ by cross-matching the QSO and SMG subsamples described above. The corresponding impact parameters $\left(R_{\perp}\right)$ are $40<R_{\perp}<300 \mathrm{kpc}$ for $z_{\mathrm{SMG}}=2.5$. The impact parameter is defined as the transverse proper distance at the redshift of the foreground SMG, which equals the angular diameter distance of the SMG multiplied by the angular separation in the sky $\left(R_{\perp}=D_{\mathrm{A}}(z) \times \theta\right)$. These QSO sightlines thus probe out to the $\sim 1.5$ virial radii of $10^{13} M_{\odot}$ halos $\left(r_{\mathrm{vir}}=200 \mathrm{kpc}\right.$ at $\left.z=2.5\right)$. Given the positional uncertainty of the Herschel $250 \mu \mathrm{m}$ sources $\left(\sigma_{\mathrm{pos}}=3\right.$ ". 4 ; see Section 4.1), the $5^{\prime \prime}$ lower limit on the angular separation is imposed as an attempt to avoid far-IR-luminous QSOs, i.e., the QSOs are the SMGs themselves. Through visual inspection of the QSO spectra, we further excluded 31 pairs whose QSOs exhibit strong broad absorption lines (this makes them unsuitable for absorption line work), have wrong redshifts, or are misclassified. Therefore, our final sample includes 199 pairs, among which $90 / 163$ QSOs have SDSS $g \leqslant 21 / 22$ (bright enough for absorption line spectroscopy). One Herschel source is probed by two QSOs at $\theta_{250}=8$ "! 0 and 18 ". 2 . No QSO probes multiple Herschel sources within $36^{\prime \prime}$, but a "single" Herschel source may consist of multiple SMGs due to the large beam size, so it is possible that a single QSO could probe multiple SMGs at different impact parameters (this is indeed the case for the last source shown in Figure 1).

\footnotetext{
17 The angular separation $\theta_{250}$ is measured as that between the Herschel $250 \mu \mathrm{m}$ position and the optical position of the QSO.
}

\section{FOLLOW-UP OBSERVATIONS}

Extensive follow-up observations are needed to perform an absorption-line analysis of SMGs. Identifying the absorption features in the spectrum of the background QSO requires a precise redshift for the foreground SMG. But the full-width-athalf-maximum (FWHM) angular resolution of Herschel-18" | $25^{\prime \prime} / 36^{\prime \prime}$ at $250 / 350 / 500 \mu \mathrm{m}$ - precludes longslit spectroscopic observations with a typical slit width of $1^{\prime \prime}$. To obtain more accurate positions and to identify blended sources, we exploited interferometer observations with the Karl G. Jansky Very Large Array (VLA). Furthermore, once the positions are determined with sub-arcsec accuracy, we need to determine the spectroscopic redshifts of the SMGs with near-IR spectrographs by targeting rest-frame optical lines that suffer less dust extinction than rest-frame UV lines. Finally, a high $\mathrm{S} / \mathrm{N}$ optical/near-UV spectrum of the background QSO is needed to detect the UV absorption lines imprinted by the diffuse medium around the SMGs. Below we describe these observations in more detail.

\subsection{SMG Identification with the VLA}

Far-IR-luminous galaxies, like our Herschel sources, are expected to be luminous in the radio wavelengths, according to the IR-radio correlation (Helou et al. 1985; Condon 1992; Ivison et al. 2010). We can thus obtain better positions for the Herschel sources by identifying the radio counterparts with interferometers. We observed $15 \mathrm{SMG}-\mathrm{QSO}$ pairs with the VLA in the $\mathrm{B}$ configuration with the $\mathrm{C}-$ band $(6 \mathrm{GHz})$ receivers (program ID: 15A-266). The sample was selected randomly from SMG-QSO pairs with QSOs brighter than $g \leqslant 21$, excluding those with $\theta_{250}>30^{\prime \prime}$. We later realized that two of the VLA targets in the HeLMS field are likely to be spurious detections because of Galactic cirrus (Clarke et al. 2016, in preparation), so we excluded them in this discussion. Table 1 lists the Herschel positions and photometry of the final VLA sample. The receivers have a total bandwidth of $4 \mathrm{GHz}$ at a central frequency of $5.9985 \mathrm{GHz}$. The targets were selected from six different extragalactic fields. To maximize the observing efficiency, we grouped the targets with their R.A. into five scheduling blocks of 0.8 to $3.1 \mathrm{hr}$. A nearby unresolved calibrator was observed every $\sim 10$ minutes. Depending on the R.A. of the targets, $3 \mathrm{C} 48,3 \mathrm{C} 286$, or 3C 295 was observed for bandpass and flux-density calibration. The entire program took $8.3 \mathrm{hr}$ of VLA time. The on-source integration time ranged from 9 to 70 minutes, allocated based on the $6 \mathrm{GHz}$ flux density estimated from fitting the Herschel photometry with the SED template of a well-studied, strongly 
Table 1

VLA-observed Herschel Sources

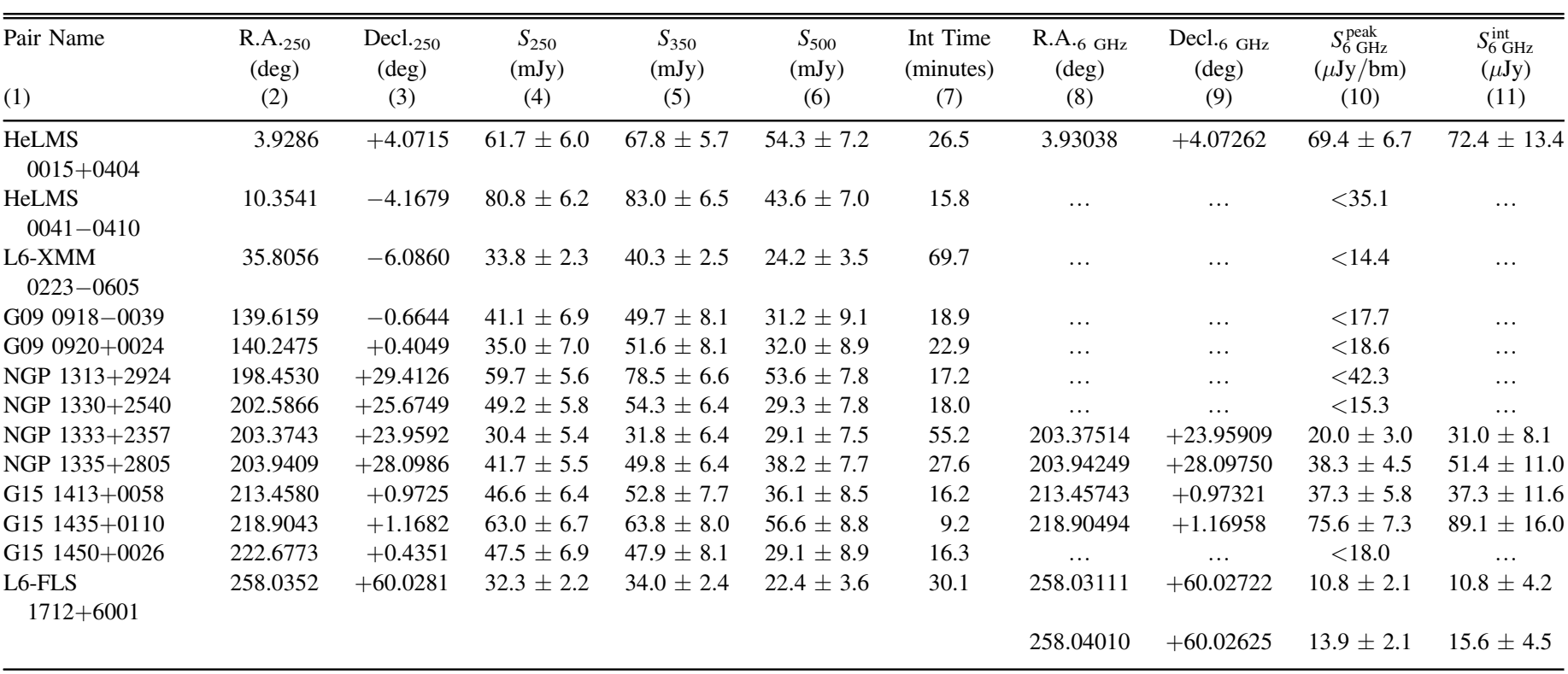

Note. L6-FLS 1712+6001 has two radio counterparts (see Figure 1); the first line shows the nominal counterpart, which is closer to the Herschel position, although slightly fainter. Columns (2-6) list the Herschel $250 \mu \mathrm{m}$ positions and the photometry at 250, 350, and $500 \mu \mathrm{m}$. Column (7) is the total VLA on-source integration time. Columns (8-9) list the positions of the radio counterparts. Columns (10-11) are the peak flux density in $\mu \mathrm{Jy} / \mathrm{bm}$ and the integrated flux density in $\mu \mathrm{Jy}$, both of which are derived by fitting an elliptical Gaussian model to the source. The uncertainty of peak flux density is given by the rms noise in the map at the source position, while the uncertainty of the integrated flux density is estimated using the formulae provided by Hopkins et al. (2003); this uncertainty includes the 1\% uncertainty in the VLA flux-density scale at $6 \mathrm{GHz}$ (Perley \& Butler 2013).

lensed SMG at $z=2.3259$ (SMM J2135-0102, aka the "Eyelash"; Swinbank et al. 2010).

The observations were calibrated using the Common Astronomical Software Applications (CASA) package (McMullin et al. 2007). We used the VLA pipeline to perform basic flagging and calibration. Additional flagging was performed whenever necessary by inspecting the visibility data. We used the self-calibration technique with bright sources within the primary beam to reduce the gain errors for four fields: HeLMS 0015+0404, HeLMS 0041-0410, L6XMM 0223-0605, and NGP 1313+2924. For imaging and deconvolution, we used the standard CASA task CLEAN with "natural" weighting to achieve the best sensitivity. The resulting restoring beams are on average 1 ". $5 \times 1$ ". 2 FWHM. The rms image noise level ranges from 2.1 to $14.1 \mu \mathrm{Jy}_{\text {beam }}{ }^{-1}$, with a mean of $6 \mu \mathrm{Jy}$ beam $^{-1}$. This measured noise is consistent with thermal noise using natural weighting of the visibility data, except for maps contaminated by the sidelobes of strong sources lying far outside the cleaned image.

We will present the results in Section 4.1, but here is a summary: The VLA detected six of the 13 SMGs; for one of those, NGP $1335+2805$, the QSO at $z=2.973$ itself was responsible for the IR emission, so we excluded it from subsequent spectroscopic observations.

\subsection{Near-IR Spectroscopy of the VLA-detected SMGs}

With the VLA positions that are accurate to $\lesssim 0$ ". 1 , we carried out a redshift survey for the five VLA-detected SMGs with near-IR spectrographs. The VLA resolved the Herschel source of L6-FLS 1712+6001 into two sources at similar brightness (Figure 1, last panel). We chose the one closer to the Herschel position as the nominal counterpart and obtained a deep near-IR spectrum at that location. It is unclear whether the two sources are physically related because the redshift of the other source remains to be determined.

We observed G15 1435+0110, L6-FLS 1712+6001, and NGP $1333+2357$ with the LUCI-1 spectrograph (LBT NIRSpectroscopic Utility with Camera and Integral-Field Unit; Seifert et al. 2003) on the Large Binocular Telescope (LBT) on 2015 April 14. We used the $200 \mathrm{l} / \mathrm{mm} H+K$ grating at $\lambda_{\mathrm{c}}=1.93 \mu \mathrm{m}$ and the N1.8 camera ( 0 ". 25 per pixel) to obtain a spectral range between 1.5 and $2.3 \mu \mathrm{m}$. The $1^{\prime \prime}$-wide $4^{\prime}$-long slit was centered on the VLA-determined SMG position, and it was aligned with the QSO in each pair to obtain the QSO spectrum simultaneously. The QSO spectrum was bright enough to serve as a useful reference for spatial alignment. The spectral resolution $(R)$ was $\sim 940$ in $H$-band and $\sim 1290$ in $K$-band. We obtained $32 \times 120 \mathrm{~s}$ exposures for each target. Between exposures, we dithered along the slit among six dithering positions distributed within $40^{\prime \prime}$. Atmospheric transparency varied dramatically during the night, and no telluric star was observed. So we used the telluric star observation from the previous night for an approximate telluric and flux calibration.

We observed HeLMS 0015+0404 and G15 1413+0058 with the Gemini near-infrared spectrograph (GNIRS; Elias et al. 2006) in the queue mode (program IDs: GN-2015B-Q46, GN-2016A-Q-41). We used the cross-dispersing prism with the $31.7 \mathrm{l} / \mathrm{mm}$ grating and the short camera to obtain a complete spectral coverage between 0.85 and $2.5 \mu \mathrm{m}$. With the 0 " 68 short slit, the spectral resolution was $R \sim 750$ across all orders. We obtained $14 \times 300 \mathrm{~s}$ exposures for HeLMS 0015 +0404 on 2015 August 9 and 12 and October 18, and $24 \times 115 \mathrm{~s}$ exposures for G15 $1413+0058$ on 2016 May 20 . We dithered by $3^{\prime \prime}$ along the $7 "$-slit between exposures.

Data reduction was carried out with a modified version of LONGSLIT_REDUCE (Becker et al. 2009) for LUCI-1 by 
Fuyan Bian (Bian et al. 2010) and a modified version of Spextool (Vacca et al. 2003; Cushing et al. 2004) for GNIRS by K. Allers (2016, private communication). These IDL packages carry out the standard data reduction steps for nearIR spectroscopy: flat-fielding, wavelength calibration, pairwise sky subtraction, residual sky removal, shifting and coadding, spectral extraction, telluric correction, and flux calibration.

We identified narrow emission lines in three of the five observed SMGs, which enabled redshift measurements. We were unable to determine the redshifts of the other two sources because we detected only the continuum emission at low $\mathrm{S} / \mathrm{N}$.

\subsection{Optical Spectroscopy of the Background QSOS}

Although previous optical spectra exist for all of the QSOs in our sample, most of them do not have the necessary wavelength coverage or sufficient $\mathrm{S} / \mathrm{N}$ for absorption line analysis. Hence, we obtained new optical spectra for the three background QSOs that were associated with the spectroscopically identified SMGs. We observed HeLMS 0015+0404 and NGP $1333+2357$ on 2016 January 9 and L6-FLS 1712 +6001 on 2015 June 13 with the Low Resolution Imaging Spectrometer (LRIS; Oke et al. 1995) on the Keck I telescope. We used the $1^{\prime \prime}$ longslit and the 560 dichroic for both runs. For the former run, we used the $600 / 4000$ Grism on the blue side $\left(R \sim 1000\right.$ at $4000 \AA$, FWHM $\left.=300 \mathrm{~km} \mathrm{~s}^{-1}\right)$ and the $600 /$ 7500 grating at $\lambda_{\mathrm{c}}=7407 \AA$ on the red side to cover 3300 to $8700 \mathrm{~A}$. For the latter run, we used the $400 / 3400 \mathrm{Grism}$ on the blue side $\left(R \sim 600\right.$ at $4000 \AA$, FWHM $\left.=500 \mathrm{~km} \mathrm{~s}^{-1}\right)$ and the $400 / 8500$ grating tilted to a central wavelength of $\lambda_{\mathrm{c}}=8300 \AA$ on the red side to cover 3300 to $10,200 \AA$. The total integration time for each source ranged between 30 and 40 minutes. Conditions were non-photometric for both nights. We obtained useful spectra for all three background QSOs.

We reduced the raw data with XIDL, an IDL data reduction package for a number of spectrographs written by two of us (JXP and JFH). The pipeline follows the standard data reduction steps and reduces the blue and red channels separately. It begins by subtracting a super bias from the raw CCD frames, tracing the slit profiles using flat fields, and deriving the 2D wavelength solution for each slit using the arcs. Then it flat-fields each slit and rejects cosmic-rays, identifies objects automatically in the slit, and builds the b-spline super sky model without rectification (Kelson 2003). After subtracting the super sky model, it performs optimal 1D extraction based on the spatial profile of the QSO (Horne 1986). Finally, it removes instrument flexure using isolated sky lines, performs heliocentric correction, and does flux calibration.

\section{RESULTS}

\subsection{Radio Detections}

We targeted the Herschel sources in 13 SMG-QSO pairs with the VLA and made six clear detections (46\% detection rate). Figure 1 shows the VLA detections, and Table 1 lists the $6 \mathrm{GHz}$ positions and flux density measurements for the detections and $3 \sigma$ upper limits on the non-detections. The VLA revealed five genuine SMG-QSO pairs (HeLMS 0015 +0404, NGP 1333+2357, G15 1413+0058, G15 1435+0110, L6-FLS 1712+6001) and one far-IR-luminous QSO (NGP 1335+2805).

The VLA-detected SMGs have $6 \mathrm{GHz}$ peak flux densities between 11 and $76 \mu \mathrm{Jy}$ with a mean of $\sim 40 \mu \mathrm{Jy}$. For these detections, there is a clear positive correlation between the observed flux densities at $6 \mathrm{GHz}$ and $500 \mu \mathrm{m}$, as expected from the IR-radio correlation convolved with a redshift distribution. The 54\% non-detection rate is not surprising given the uncertainties in the predicted $6 \mathrm{GHz}$ flux density and the fact that the sensitivity of radio data may deviate substantially from the theoretical prediction because of confusion sources and weather.

The offsets between the Herschel positions and the VLA positions range between 2 !' 7 and $7 ! .9$. The maximum offset is comparable to the estimated $2 \sigma$ positional uncertainty of Herschel $250 \mu \mathrm{m}$ selected catalogs: $\sigma_{\text {pos }}=\sqrt{2} \sigma_{\text {R.A. }}=$ $0.93 \times \mathrm{FWHM} / \mathrm{SNR}=3$ ! 4 for $\mathrm{FWHM}=18$ !" 1 and $\mathrm{SNR}=5$ (Ivison et al. 2007; Smith et al. 2011). This indicates that a fraction of the "pairs" that have small angular separations $\left(\theta_{250} \lesssim 8^{\prime \prime}\right)$ could be single sources, i.e., QSOs that are far-IR-luminous. In our VLA-detected sample, NGP $1335+2805$, with $\theta_{250}=6$ ". 5 , is the only such case (see the third panel of Figure 1). The VLA position agrees with the optical QSO position within 0 ". 1 , which is well within the cross-band astrometry accuracy of $\sim 0$ ". 3 (SDSS+VLA; e.g., Ivezić et al. 2002). But on the other hand, the SMG in L6-FLS $1712+6001$ is a separate source from the QSO, despite its even smaller $250 \mu \mathrm{m}$ separation $\left(\theta_{250}=5\right.$ !" 4$)$. We thus chose not to exclude pairs with $5^{\prime \prime}<\theta_{250}<8^{\prime \prime}$. The far-IR-luminous QSOs in our sample are interesting in their own right, and they will be discussed elsewhere.

\subsection{Physical Properties of the SMGs}

Estimating the intrinsic properties of the VLA-detected Herschel sources requires knowledge of their spectroscopic redshifts. We obtained deep near-IR spectra for the five VLA counterparts whose spectroscopic redshifts were previously unknown. We detected $\mathrm{H} \alpha$ and [N II] lines from three of the five sources, enabling accurate determination of the spectroscopic redshifts (Figure 2 and Table 2). The [N II]/H $\alpha$ line ratios are consistent with the range observed in typical SMGs $(0.1 \lesssim[\mathrm{N} \mathrm{II}] / \mathrm{H} \alpha \lesssim 1.4 ;$ Swinbank et al. 2004). The two remaining sources, G15 1413+0058 and G15 1435+0110, show near-IR continuum emission without detectable emission lines or stellar absorption features. It is possible that the emission lines either fall into one of the telluric absorption bands or are simply outside of the spectral range. Our redshift success rate is thus $60 \%$, comparable to those of previous redshift surveys of SMGs in the optical range (e.g., Chapman et al. 2005; Casey et al. 2011).

With the photometry from Herschel and the VLA and spectroscopic redshifts, we can estimate the total rest-frame 8 $-1000 \mu \mathrm{m}$ luminosity $\left(L_{\mathrm{IR}}\right)$ and the IR-to-radio luminosity ratio $\left(q_{\mathrm{IR}}\right)$. The results are listed in Table 2. We fit the Herschel photometry with a modified blackbody at the spectroscopic redshift, with $\beta$ fixed to 1.5 . We found that they have $L_{\mathrm{IR}}>10^{12} L_{\odot}$ and $\mathrm{SFR}_{\mathrm{IR}}=470-1500 M_{\odot} \mathrm{yr}^{-1}$. The $\mathrm{SFR}$ was estimated from $L_{\mathrm{IR}}$ using the calibration of Murphy et al. (2011) for a Kroupa (2002) initial mass function:

$$
\mathrm{SFR} / M_{\odot} \mathrm{yr}^{-1}=1.5 \times 10^{-10} L_{\mathrm{IR}} / L_{\odot} \cdot
$$

The extrapolated $850 \mu \mathrm{m}$ flux densities are in the range $7 \leqslant S_{850} \leqslant 18 \mathrm{mJy}$, confirming that they are SMGs. 

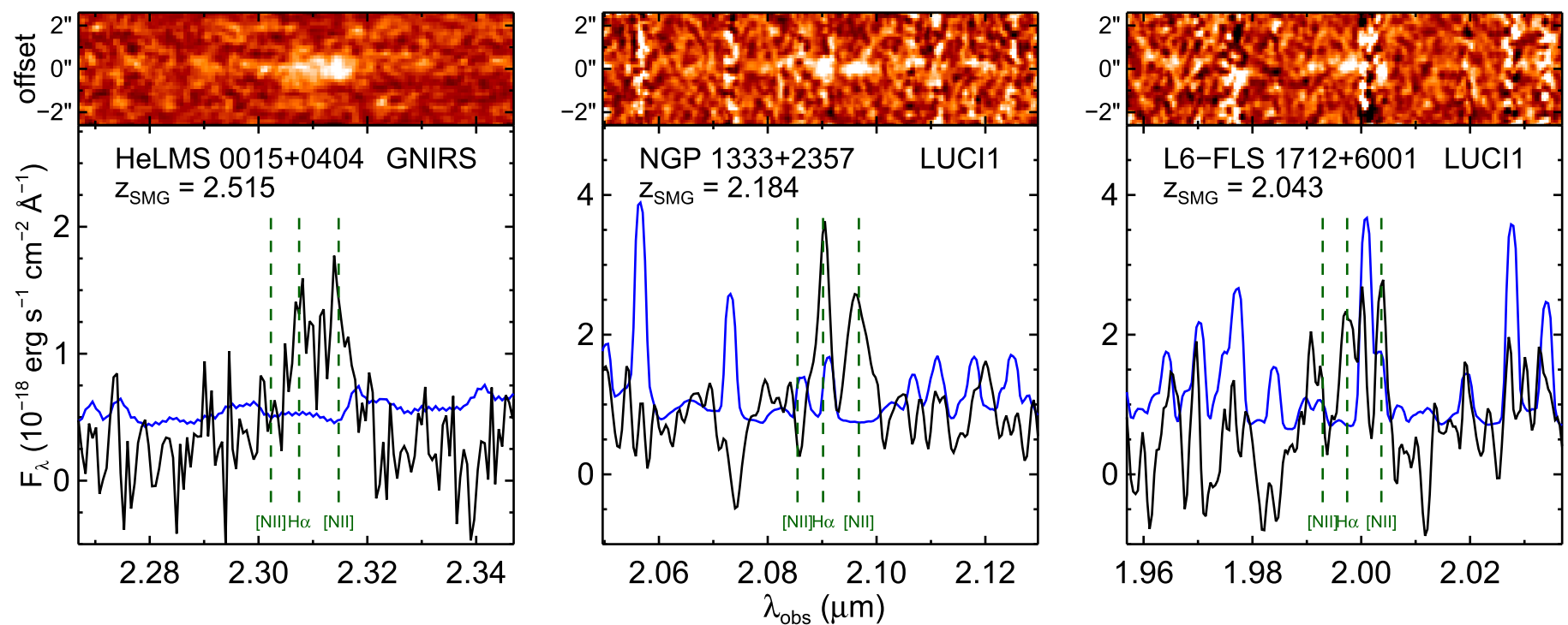

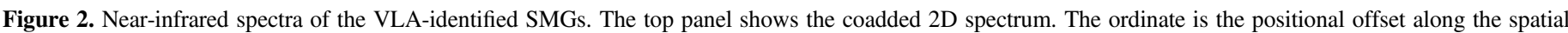

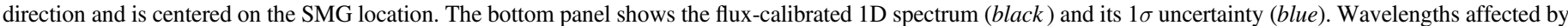
strong sky lines show large errors. The dashed lines indicate the redshifted $\mathrm{H} \alpha \lambda 6563$ and [N II] $\lambda \lambda 6548,6583$ lines.

The IR-to-radio luminosity/flux ratio was estimated using the following equation (Helou et al. 1985; Ivison et al. 2010):

$$
q_{\mathrm{IR}}=\log \frac{S_{\mathrm{IR}} / 3.75 \times 10^{12} \mathrm{~W} \mathrm{~m}^{-2}}{S_{1.4 \mathrm{GHz}} / \mathrm{W} \mathrm{m}^{-2} \mathrm{~Hz}^{-1}}
$$

where $S_{\mathrm{IR}}$ is the rest-frame integrated $8-1000 \mu \mathrm{m}$ flux and $S_{1.4 \mathrm{GHz}}$ is the rest-frame $1.4 \mathrm{GHz}$ flux density. ${ }^{18}$ The former is from the modified blackbody fit to the IR SED, and the latter is converted from the observed $6 \mathrm{GHz}$ flux densities assuming a typical synchrotron slope $\left(S_{\nu} \propto \nu^{-0.8}\right)$. The three $\mathrm{SMGs}$ show $2.1 \leqslant q_{\mathrm{IR}} \leqslant 2.6$, consistent with the observed radio-IR correlation for Herschel sources $\left(q_{\mathrm{IR}}=2.4 \pm 0.5\right.$; Ivison et al. 2010). This indicates that our sample is not biased to radio-loud AGNs despite being radio selected.

\subsection{Absorption Line Systems}

To compare our results with previous results on the CGM of other high-redshift galaxies, we needed to characterize the covering factor of optically thick gas clouds around SMGs (i.e., Lyman limit systems [LLSs] with column densities of neutral hydrogen $N_{\mathrm{H} \mathrm{I}}>10^{17.2} \mathrm{~cm}^{-2}$ ). We searched for Ly $\alpha$ absorbers near the spectroscopic redshifts of the foreground SMGs and classified the Ly $\alpha$ absorbers in the QSO spectra following the procedure used in the QPQ study (e.g., Prochaska et al. 2013b). We classified the absorbers as optically thick (i.e., LLSs), optically thin, or ambiguous based on the Ly $\alpha$ equivalent widths (EWs) and the presence of associated metal transitions.

We first identified H I Ly $\alpha$ absorption due to the SMGs in the QSO spectra within $\pm 600 \mathrm{~km} \mathrm{~s}^{-1}$ of the systemic redshifts of the SMGs. The search window was chosen because the escape velocity is $610 \mathrm{~km} \mathrm{~s}^{-1}$ at the virial radius $\left(R_{\mathrm{vir}}=230\right.$ $\mathrm{kpc}$ ) of a $10^{13} M_{\odot}$ dark matter halo at $z=2$ (Navarro et al. 1996; Bullock et al. 2001). Strong Ly $\alpha$ absorption lines were detected in all three systems (Figure 3). We report their rest-frame EWs $\left(W_{\mathrm{Ly} \alpha}\right)$, the updated angular separations based

\footnotetext{
18 This flux ratio is equivalent to the luminosity ratio defined in Kovács et al. (2006).
}

on the VLA positions $\left(\theta_{6 \mathrm{GHz}}\right)$, and the impact parameters $\left(R_{\perp}\right)$ in Table 2. As we have found in our earlier QSO absorption line studies, systematic error associated with continuum placement and line blending generally dominates the statistical error of the QSO spectra. So we estimated the error of $W_{\mathrm{Ly} \alpha}$ assuming a $10 \%$ error in the continuum placement. From our best-fit Gaussians to the absorption profiles (shown in Figure 3), we found that all three systems show strong $\mathrm{Ly} \alpha$ absorption lines with $W_{\mathrm{Ly} \alpha}=1.7-2.9 \AA$. Note that when $z_{\mathrm{QSO}}-z_{\mathrm{SMG}} \gtrsim 0.5$, the H I Ly $\alpha$ absorption from the SMG may lie within the QSO Ly $\beta$ forest. Unfortunately, all three pairs fall in this category, so contamination from $\operatorname{Ly} \beta$ lines from systems in the $\operatorname{Ly} \alpha$ forest may be a concern. However, it turned out that $\operatorname{Ly} \beta$ contamination is not a serious issue for these systems. Because we assumed the detected Ly $\alpha$ absorption to be $\operatorname{Ly} \beta$, we searched for the corresponding Ly $\alpha$ lines in each spectrum but did not find any.

Given the absorption profiles of the Ly $\alpha$ line, we then searched for the associated metal transitions commonly observed in optically thick absorption systems (e.g., Prochaska et al. 2015): Si II $\lambda 1260,1304,1527$, O I $\lambda 1302$, C II $\lambda 1335$, Si IV $\lambda 1394,1403, \quad$ C IV $\lambda 1548,1551, \quad$ Fe II $\lambda 1608,2383,2600$, Al II $\lambda 1671$, and $\mathrm{Mg}$ II $\lambda 2796,2804$. However, none of these metal transitions were convincingly detected in any of our systems. In the following, we discuss the systems individually and provide our classifications:

1. HeLMS 0015+0404. This system shows an H I Ly $\alpha$ absorption at $+500 \mathrm{~km} \mathrm{~s}^{-1}$ with $W_{\mathrm{Ly} \alpha}=2.0 \pm 0.2 \AA$ and intrinsic ${ }^{19} \mathrm{FWHM}=430 \mathrm{~km} \mathrm{~s}^{-1}$ (Figure 3 top panels). The $\mathrm{EW}$ gives an upper limit on the $\mathrm{H}$ I column density at $\log \left(N_{\mathrm{H}}\right) \lesssim 18.9 \pm 0.1 \mathrm{~cm}^{-2}$. The column density is estimated from the theoretical curve of growth where $W_{\mathrm{Ly} \alpha}=7.3\left(N_{\mathrm{Hi}} / 10^{20} \mathrm{~cm}^{-2}\right)^{0.5} \AA$ for $N_{\mathrm{H} \mathrm{I}}>10^{18}$ $\mathrm{cm}^{-2}$, the regime where the relation is insensitive to the Doppler $b$-parameter (e.g., Mo et al. 2010, Section 16.4.4). This is an upper limit because it assumes that the

\footnotetext{
19 Instrumental broadening has been deconvolved.
} 
Table 2

Properties of the Spectroscopically Confirmed SMG-QSO pairs

\begin{tabular}{|c|c|c|c|c|c|c|c|c|c|c|}
\hline Pair Name & $\begin{array}{c}\text { SMG } \\
(\mathrm{J} 2000)\end{array}$ & $z_{\mathrm{SMG}}$ & $\begin{array}{c}\log \left(L_{\mathrm{IR}}\right) \\
\left(L_{\odot}\right)\end{array}$ & $q_{\mathrm{IR}}$ & $\begin{array}{c}\text { QSO } \\
(\mathrm{J} 2000)\end{array}$ & $z_{\mathrm{QSO}}$ & $\begin{array}{c}\theta_{6 \mathrm{GHz}} \\
\left({ }^{\prime \prime}\right)\end{array}$ & $\begin{array}{c}R_{\perp} \\
(\mathrm{kpc})\end{array}$ & $\begin{array}{c}W_{\mathrm{Ly} \alpha} \\
(\AA)\end{array}$ & $\begin{array}{l}\text { Optical Depth } \\
\text { Classification }\end{array}$ \\
\hline HeLMS 0015+0404 & J001543.29+040421.4 & 2.515 & 13.1 & 2.1 & J001542.31+040433.5 & 3.256 & 19.0 & 157 & $2.0 \pm 0.2$ & ambiguous \\
\hline NGP $1333+2357$ & $\mathrm{~J} 133330.03+235732.7$ & 2.184 & 12.6 & 2.3 & $\mathrm{~J} 133330.36+235709.9$ & 3.108 & 23.3 & 198 & $1.7 \pm 0.2$ & ambiguous \\
\hline L6-FLS 1712+6001 & $\mathrm{J} 171207.47+600138.0$ & 2.043 & 12.6 & 2.6 & $\mathrm{~J} 171209.00+600144.4$ & 2.821 & 13.1 & 112 & $2.9 \pm 0.5$ & optically thin \\
\hline
\end{tabular}

absorption is dominated by a single component, while line blending is likely to be in the forest. There is putative C II absorption at $+400 \mathrm{~km} \mathrm{~s}^{-1}\left(W_{\mathrm{CII}}=1.2 \AA\right)$; but it is likely a false identification of a $\operatorname{Ly} \alpha$ forest line, because it is misaligned in velocity with the $\operatorname{Ly} \alpha$ absorption and none of the strong metal lines in the "clean" region outside of the Lyman forests is detected (e.g., Si II, C IV, Fe II, Al II). To assess our sensitivity to these strong metal lines in a typical LLS, we overlay the expected absorption profiles on top of the observed spectra in Figure 3. The model absorption lines are smoothed to the spectral resolution and have the average EWs in LLSs around $z \sim 2$ QSOs: $0.6 \AA$ for $\mathrm{C}$ II and $0.3 \AA$ for the other lines (Prochaska et al. 2013a). It is clear that the $\mathrm{S} / \mathrm{N}$ of our spectra are high enough to detect the metal lines from a typical LLS when the lines lie outside of the Lyman forest. Without detections of strong metal lines and/or the damping wings in $\operatorname{Ly} \alpha$, we cannot conclude that the absorber is optically thick based on current data because the high $W_{\mathrm{Ly} \alpha}$ could be due to line blending. We conservatively classify this system as ambiguous, even though the non-detection of strong metal lines strongly suggests that it is an optically thin absorber, because fewer than $5 \%$ of optically thick absorbers have no detection of a metal transition (Prochaska et al. 2015).

2. NGP $1333+2357$. This system shows an $\mathrm{HI} \mathrm{Ly} \alpha$ absorption at $-34 \mathrm{~km} \mathrm{~s}^{-1}$ with $W_{\mathrm{Ly} \alpha}=1.7 \pm 0.2 \AA$ and intrinsic $\mathrm{FWHM}=260 \mathrm{~km} \mathrm{~s}^{-1}$ (Figure 3 middle panels). Similar to that of HeLMS 0015+0404, the EW gives an upper limit on the $\mathrm{HI}$ column density at log $\left(N_{\mathrm{H} \mathrm{I}}\right) \lesssim 18.8 \pm 0.1 \mathrm{~cm}^{-2}$. We observed putative absorption around the expected locations of C II, Si IV, Si II, and CIV. But all of these lines lie within the Ly $\alpha$ forest, and their velocity profiles are distinctly different from that of the Ly $\alpha$ line, indicating that these putative metal absorptions are spurious. The Fe II and Al II lines are outside of the Lyman forests, but the expected absorption lines are not detected. As in the previous case, we conservatively classify this system as ambiguous.

3. L6-FLS $1712+6001$. There is a broad but shallow H I Ly $\alpha$ absorption line at $+200 \mathrm{~km} \mathrm{~s}^{-1}$ with $W_{\mathrm{Ly} \alpha}=2.9 \pm 0.5 \AA$ and intrinsic $\mathrm{FWHM}=950 \mathrm{~km}$ $\mathrm{s}^{-1}$ (Figure 3 bottom panels). The EW gives an upper limit on the $\mathrm{H}$ I column density at $\log \left(N_{\mathrm{H}}\right) \lesssim 19.2 \pm 0.2$ $\mathrm{cm}^{-2}$. But this system is most likely optically thin because the line center drops only to $38 \pm 4 \%$ of the continuum intensity (i.e., it does not go line black like the other two systems). Applying instrumental broadening and pixel sampling to theoretical Voigt profiles, we found that the line center depth places a much stronger limit on the H I column density than the EW: log $\left(N_{\mathrm{H} \mathrm{I}}\right) \lesssim 17.5-15.8$ at $b=20-30 \mathrm{~km} \mathrm{~s}^{-1}$, the range of $b$ parameters observed in $\mathrm{HI}$ absorbers at $z \sim 2-3$ (e.g., $\mathrm{Hu}$ et al. 1995; Rudie et al. 2012). As in the previous cases, we observed putative metal absorption inside the Lyman forests (e.g., O I, C II, Si II, and Si IV) but no metal absorption outside of the forests (e.g., C IV, Fe II, Al II, and $\mathrm{Mg} \mathrm{II}^{20}$ ). Note that the weak $\mathrm{CIV}$ absorption is blueshifted by more than $200 \mathrm{~km} \mathrm{~s}^{-1}$ from the Ly $\alpha$ line center. Considering the strict limit on the H I column density and the absence of metal lines, we classify this system as optically thin.

In summary, we have identified one clearly optically thin case and two ambiguous cases with three QSO sightlines covering impact parameters within $100<R_{\perp}<200 \mathrm{kpc}$ around SMGs at $2.0<z<2.6$. The two ambiguous cases are also likely to be optically thin, given the non-detection of any metal transitions.

\subsection{Comparison with the CGM Around QSOs}

For background QSO sightlines probing the CGM of $z \sim 2-3$ foreground QSOs, one observes H I absorbers optically thick at the Lyman limit $\gtrsim 60 \%$ of the time. The high $\mathrm{H}$ I covering factor extends to at least the expected virial radius of $\sim 160 \mathrm{kpc}$ (Hennawi et al. 2006a; Prochaska et al. 2013a, 2013b). In Figure 4, we compare (1) the SMG sample distribution with the QSOs from the QPQ project and (2) the covering factor of optically thick H I gas around SMGs with that around QSOs. The two samples overlap in the plane of foreground redshift versus impact parameter: the SMGs cover the same redshift range as the QSOs in the intermediate impact parameter range between 100 and $200 \mathrm{kpc}$. We calculated the $1 \sigma$ binomial confidence intervals of the optically thick fraction using the quantiles of the beta distribution (Cameron 2011). Because there is no clearly optically thick absorber among the three systems we analyzed, the $1 \sigma$ confidence interval of our covering factor is $4.2 \%-36.9 \%$ for a non-detection in a sample of three. For comparison, we considered all of the QSO sightlines with $100<R_{\perp}<200 \mathrm{kpc}$ from the QPQ project (Prochaska et al. 2013b), and we found the optically thick covering factor is $64_{-9}^{+7} \%$ for 21 clearly optically thick systems among 33 systems. Therefore, despite our small sample, the upper bound of our $1 \sigma$ confidence interval is $3 \sigma$ below the bestestimated covering factor around QSOs at the same ranges of redshifts and impact parameters.

Note that although our analysis may appear to have limitations based on the classification of two of the SMG absorbers as ambiguous, these same limitations also apply to the QPQ analysis and are inherent to any attempt to classify absorbers using low- or moderate-resolution spectra without coverage of the H I Lyman limit (at $912 \AA$ in the rest-frame). As such, we followed exactly the same procedure for absorber classification as in the QPQ studies, enabling a direct comparison to that work. Our data show that clear optically

\footnotetext{
${ }^{20}$ Because of the wider spectral coverage and the lower foreground redshift, this is the only object where we have coverage of the $\mathrm{Mg}$ II $\lambda 2796,2804$ lines.
} 

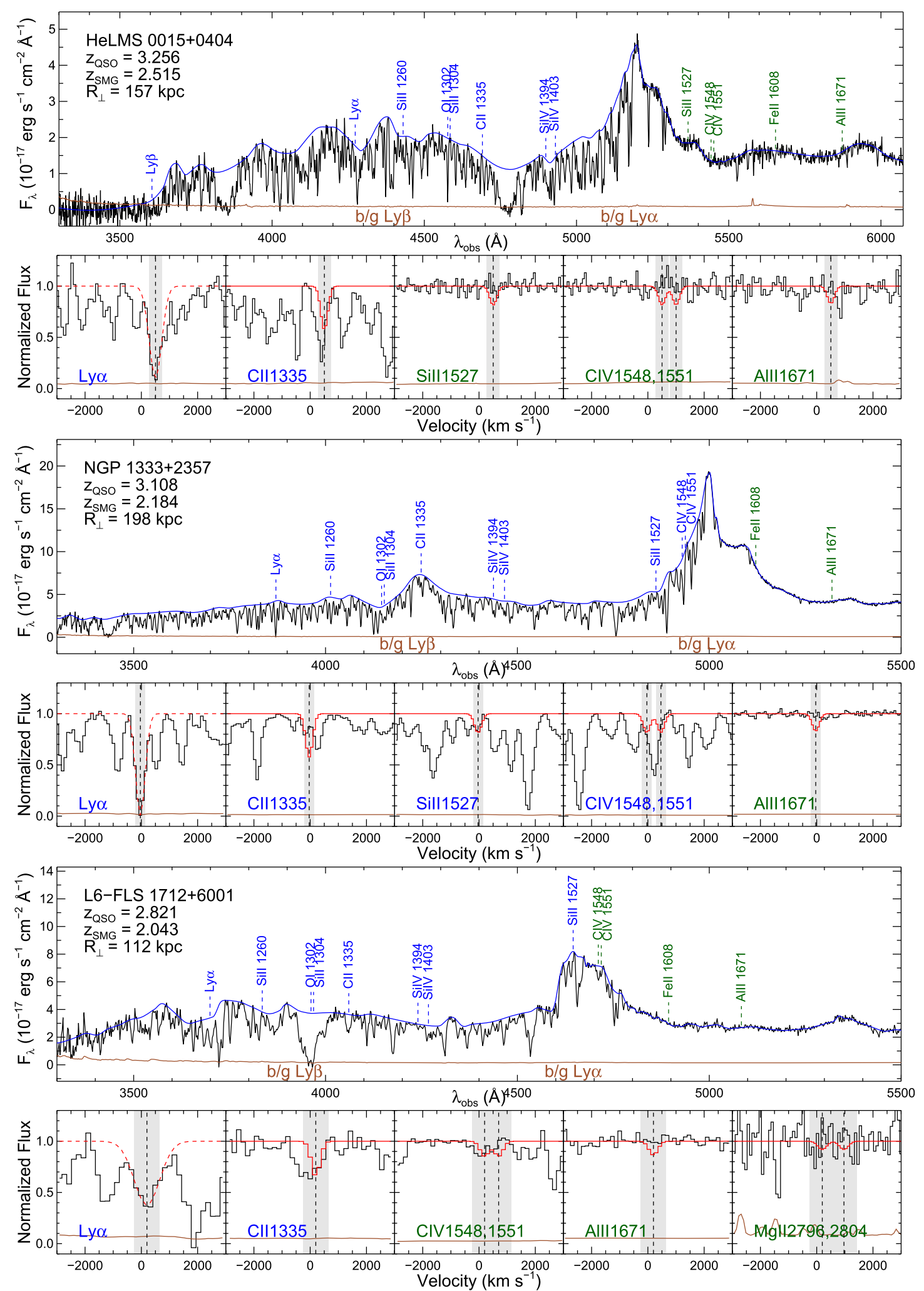

Figure 3. Keck optical spectra of background (b/g) QSOs probing foreground SMGs. The black curve shows the observed flux-calibrated spectrum, while the blue curve shows the continuum model used to normalize the spectrum. The expected locations of absorption lines due to the foreground SMG are marked in blue and green for lines inside and outside the Lyman forests, respectively. Below the full spectrum, we also show the velocity profiles for the $\mathrm{H}$ I Ly $\alpha$ and a number of common metal absorption lines. All panels show the region $\pm 3000 \mathrm{~km} \mathrm{~s}^{-1}$ around the systemic redshifts of the foreground SMGs. On the first panel, we show a Gaussian fit to the strongest H I Ly $\alpha$ absorption line within our search window of $\pm 600 \mathrm{~km} \mathrm{~s}^{-1}$. In other panels, we overlay the metal line profiles of a typical LLS, using the average observed EWs ( $0.6 \AA$ for $\mathrm{C}$ II and $0.3 \AA$ for other lines; Prochaska et al. 2013a). The vertical dashed line shows the centroid velocity, and the gray shaded region highlights the $\pm 1 \sigma$ width of the best-fit Gaussian. Both components of the C IV and Mg II doublets are highlighted. 


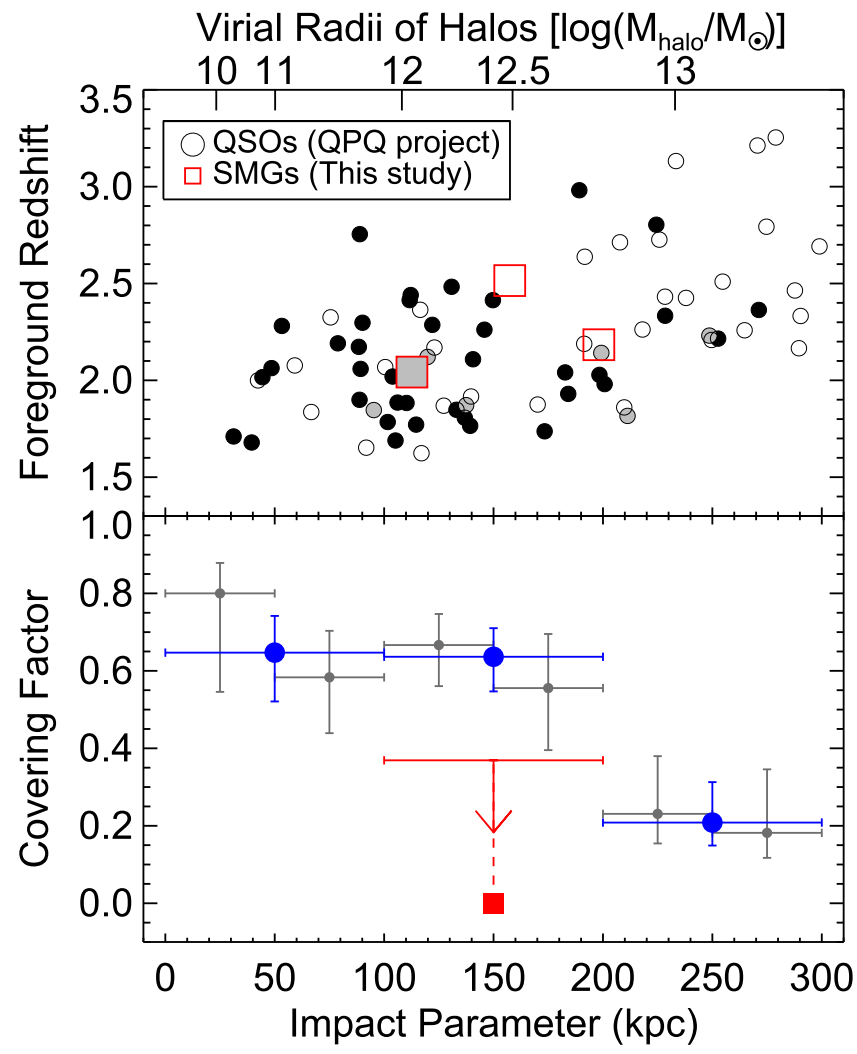

Figure 4. Top: sample distributions in foreground redshift vs. impact parameter. The red squares and the black circles show the SMGs from this study and the QSOs from the QPQ survey (Prochaska et al. 2013b), respectively. The black-filled, gray-filled, and open symbols correspond to systems that are optically thick, optically thin, and ambiguous, respectively. The top axis marks the virial radii for a range of dark matter halos at $z=2$. Bottom: the covering factor of optically thick gas around SMGs (red square with a downward arrow: our $1 \sigma$ confidence interval for a non-detection in a sample of three) vs. impact parameter, compared to that around QSOs from the QPQ survey (blue/gray circles with error bars; Prochaska et al. 2013b). The blue and gray circles are estimates of the covering factor based on $100 \mathrm{kpc}$ wide bins and $50 \mathrm{kpc}$ wide bins, respectively. Our sample probes similar ranges of redshifts and impact parameters as the QPQ QSOs but shows less optically thick absorbers.

thick cases appear much less frequently around SMGs than around coeval QSOs. However, if we treat the ambiguous cases in both samples as if they were optically thick absorbers, then the difference in the optically thick covering factor becomes smaller because of the high number of ambiguous cases in the SMG sample: the covering factor around SMGs increases to $67_{-28}^{+15} \%$ ( 2 out of 3 systems), while the covering factor around $z \sim 2$ QSOs increases to $91_{-8}^{+3} \%$ (30 out of 33 systems) within $100<R_{\perp}<200 \mathrm{kpc}$.

There are some minor differences between our analysis and the QPQ analysis. But they are unlikely to affect our result. First, the bulk of the QPQ dataset (Prochaska et al. 2013a) utilized background QSO spectra with moderate resolution $(R \sim 2000)$. This is a factor of two higher than the resolution we used for the QSO spectra probing two of our SMGs $(R \sim 1000)$, whereas in L6-FLS $1712+6001$ our spectrum has $R \sim 600$. But the lower resolution of our spectra does not play a significant role in the resulting classification of our absorbers, since the typical strength of the metal lines seen in the optically thick systems would still be easily detectable at $R \sim 1000$, and the one system that we observed at $R \sim 600$ is most likely optically thin, since it does not go line black. Another significant difference is whereas Prochaska et al. (2013a) restricted analysis to only those foreground QSOs with redshifts lying within the Ly $\alpha$ forest of the background QSO, we included foreground SMGs landing in the Ly $\beta$ forest, owing to the larger redshift separations between the SMGs and the QSOs in our sample. This is unlikely to impact our deduced covering factor in Figure 4 though. For example, earlier QPQ studies (Hennawi et al. 2006b; Hennawi \& Prochaska 2007, 2013) also considered absorbers lying in the $\operatorname{Ly} \beta$ forest, and the covering factors deduced there are consistent with that derived in Prochaska et al. (2013a). Furthermore, in all of the three SMG-QSO pairs shown in Figure 3, multiple metal lines that are strong in optically thick absorbers (i.e., Si II $\lambda 1527$, $\mathrm{C} \operatorname{IV} \lambda \lambda 1548,1551, \mathrm{Al}$ III $\lambda 1671$, and $\mathrm{Mg}$ II $\lambda \lambda 2796,2804)$ land in the clean regions outside of the Lyman forest but remain undetected. Finally, whereas QPQ searched for absorbers within a velocity window of $\pm 1500 \mathrm{~km} \mathrm{~s}^{-1}$ owing to the large errors in QSO redshifts, we adopted a $\pm 600 \mathrm{~km} \mathrm{~s}^{-1}$ search window. We were able to consider a smaller velocity interval because our SMG redshifts derived from narrow rest-frame optical lines are much more accurate, and this interval was instead chosen to encompass the typical virial motions in a $10^{13} M_{\odot}$ dark matter halo. The larger velocity interval used in QPQ formally implies a higher level of contamination from physically unassociated absorbers. But the contamination is estimated to be at only the $\sim 3 \%$ level for a $\pm 1500 \mathrm{~km} \mathrm{~s}^{-1}$ window (Prochaska et al. 2013a; see their Figure 10 and Table 7). This is too small to be responsible for the difference in the optically thick covering factor that we observed between QSOs and SMGs.

\section{SUMMARY AND CONCLUSIONS}

Motivated by the unique properties of SMGs and their purported evolutionary link to high-redshift QSOs and today's massive ellipticals, we have started a project to use QSO absorption line spectroscopy to probe the diffuse cool H I gas in the CGM of SMGs. This work requires a sample of projected SMG-QSO pairs, which are extremely rare. Wide-area submillimeter surveys are needed to compile such a sample. Thanks to the advent of Herschel, we have identified 163 SMG -QSO pairs with bright $z>2.5$ QSOs $\left(g_{\mathrm{QSO}}<22\right)$ and angular separations between $5^{\prime \prime}$ and $36^{\prime \prime}$ from a suite of widearea Herschel surveys and spectroscopic QSO surveys.

Extensive follow-up observations are required to carry out the absorption line study. To allow slit spectroscopy, the first stage is to use an interferometer to pin down the positions of the Herschel sources. This paper focuses on a subsample of 13 SMG-QSO pairs that were observed with the VLA in C-band. With an average integration time of 25 minutes per source, the VLA detected sources within the Herschel beam in six fields. One of the six SMG-QSO pairs turns out to be a far-IRluminous QSO at $z=2.973$, while the Herschel source in another pair turns out to be a pair of SMGs. Hence, we effectively identified six SMG-QSO pairs from observations of 13 fields (46\%). The second stage is to measure the spectroscopic redshifts of the SMGs. We observed five of the six VLA-identified SMG-QSO pairs with near-IR spectroscopy. We were able to determine the redshifts for three of the five SMGs $(60 \%)$ from the redshifted $\mathrm{H} \alpha$ and [N II] lines. The remaining two sources show only a featureless continuum in the near-IR windows, making it impossible to determine an accurate redshift. The last stage is to obtain optical 
spectroscopy of the QSOs once it is confirmed that the QSOs are in the background of the SMGs. Because we selected only the brightest QSOs, the success rate of this step approaches $100 \%$. Because of the low success rates of the first two follow-up stages, only $\sim 23 \%$ of the initial sample (i.e., three SMG-QSO pairs out of 13) is spectroscopically confirmed and is suitable for final absorption line study. Our main findings are as follows:

1. The near-IR spectra of the five VLA-detected sources were all detected in hour-long integrations with 8-meter telescopes, although only three of those show emission lines that yielded accurate spectroscopic redshifts. Since we positioned the slits on the VLA positions, this result shows that the spatial offset, if any, between the near-IR and the radio counterparts is less than an arcsec (the slit width), consistent with the finding from differential lensing in strongly lensed sources (e.g., Fu et al. 2012).

2. The VLA-identified Herschel "350 $\mu$ m peakers" at $2.0<z<2.6$ are similar to SMGs selected at longer wavelengths (i.e., $850 \mu \mathrm{m}$ to $1 \mathrm{~mm}$ ) in terms of the [N II]/ $\mathrm{H} \alpha$ ratio (i.e., gas metallicity), the IR luminosity (i.e., $\mathrm{SFR}$ ), and the IR-to-radio luminosity ratio (i.e., radio excess due to the AGN). The VLA-identified SMGs are optically faint and unbiased to radio-loud AGNs, so they indeed represent a galaxy population distinct from the optical selected QSOs and the Lyman break galaxies (LBGs) in the same redshift range.

3. Strong $\mathrm{H} \mathrm{I} \mathrm{Ly} \alpha$ absorption was found in the background QSO spectra for all of the three spectroscopically confirmed SMG-QSO pairs with impact parameters of $100<R_{\perp}<200 \mathrm{kpc}$. Here we adopted a much narrower search window $\left( \pm 600 \mathrm{~km} \mathrm{~s}^{-1}\right)$ than the QPQ study, further reducing the level of contamination from physically unrelated clouds. However, none of the three absorption line systems seems optically thick at the Lyman limit (i.e., LLSs with $N_{\mathrm{H}}>10^{17.2} \mathrm{~cm}^{-2}$ ), in contrast to the $\sim 60 \%$ covering factor of LLSs around QSOs from the QPQ study despite similar data quality, foreground redshifts, and impact parameters.

Our comparison thus suggests either that SMGs do not have a substantial neutral gas reservoir in their halos that could potentially fuel a prolonged star formation phase or that SMGs inhabit $\sim 10^{12} M_{\odot}$ halos, so that our sightlines have yet to probe inside their virial radii. If the latter, their halos are comparable to those of LBGs. Rudie et al. (2012) found an optically thick covering factor of $30 \pm 14 \%$ around LBGs at $z \sim 2.3$ and $R_{\perp}<90 \mathrm{kpc}$. Note that this is $\sim 2$ times lower than that of coeval QSOs and is consistent with the $1 \sigma$ confidence interval that we were able to place for the SMGs. On the other hand, the difference in the optically thick H I covering factor between SMGs and QSOs casts doubt on the evolutionary link between the two populations, unless AGN outflows can somehow affect the physical state of gas at hundreds of kpc scales within its short lifetime.

Our final conclusion is limited by the small sample size. To enable a more robust comparison with previous absorption-line studies, we badly need to increase the effective yield of our survey from the current level of $\sim 23 \%$. In a future publication, we will present observations with the Atacama Large Millimeter/Submillimeter Array (ALMA) to pinpoint the positions of the Herschel sources in the SMG-QSO pairs.
Observing at a wavelength $(870 \mu \mathrm{m})$ much closer to the selection wavelengths $(250-500 \mu \mathrm{m})$, we expect to double the detection rate with integration times of just several minutes per source. The Herschel sources in our sample are too faint to allow a quick $\mathrm{CO}$ redshift search with ALMA (e.g., the survey of strongly lensed SMGs by Weiß et al. 2013), but spectrographs on large optical telescopes covering the full optical+IR range at a moderate spectral resolution $(R \gtrsim 2000)$ will greatly increase the redshift search range and decrease the areas blinded by strong airglow lines.

We thank F Bian and $\mathrm{K}$ Allers for providing the data reduction packages, D Ludovici for helping with the VLA data calibration of L6-XMM 0223-0605, and the anonymous referee for comments that helped improve the presentation of the paper. We acknowledged M. Lau for her assistance in obtaining the Keck observations. The National Radio Astronomy Observatory is a facility of the National Science Foundation (NSF) operated under cooperative agreement by Associated Universities, Inc. Support for this work was provided by the NSF through award GSSP SOSPA3-016 from the NRAO. H.F. acknowledges support from NSF grant AST1614326, NASA JPL award 1495624, and funds from the University of Iowa. J.X.P. acknowledges support from NSF grants AST-1010004, AST-1109452, AST-1109447, and AST1412981. A.C. acknowledges support from NSF grant AST1313319 and NASA grants NNX16AF39G and NNX16AF38G. D.K. acknowledges support from NSF grant AST-1412153 and funds from the University of California, San Diego. Z.Y.Z. acknowledges support from the European Research Council in the form of the Advanced Investigator Programme, 321302, COSMICISM. The Herschel-ATLAS is a project with Herschel, which is an ESA space observatory with science instruments provided by European-led Principal Investigator consortia and with important participation from NASA. The H-ATLAS website is http://www.h-atlas.org/. The US participants acknowledge support from the NASA Herschel Science Center/JPL. The LBT is an international collaboration among institutions in the United States, Italy, and Germany. The LBT Corporation partners are the University of Arizona, on behalf of the Arizona University System; Istituto Nazionale di Astrofisica, Italy; LBT Beteiligungsgesellschaft, Germany, representing the Max-Planck Society, the Astrophysical Institute Potsdam, and Heidelberg University; the Ohio State University; and the Research Corporation, on behalf of the University of Notre Dame, the University of Minnesota, and the University of Virginia. Some of the data presented herein were obtained at the W.M. Keck Observatory, which is operated as a scientific partnership between the California Institute of Technology, the University of California, and the National Aeronautics and Space Administration. The Observatory was made possible by the generous financial support of the W.M. Keck Foundation. Our data are based on observations obtained at the Gemini Observatory, which is operated by the Association of Universities for Research in Astronomy, Inc., under a cooperative agreement with the NSF on behalf of the Gemini partnership: the NSF (United States), the National Research Council (Canada), CONICYT (Chile), Ministerio de Ciencia, Tecnología e Innovación Productiva (Argentina), and Ministério da Ciência, Tecnologia e Inovação (Brazil). The authors wish to recognize the very significant cultural role that the summit of Mauna Kea has always had within the 
indigenous Hawaiian community, who hold this feature in deep reverence. We are most fortunate to have the opportunity to conduct observations from this mountain.

Facilities: Herschel, Sloan, VLA, Keck/LRIS, LBT/ LUCI-1, Gemini/GNIRS.

\section{REFERENCES}

Alam, S., Albareti, F. D., Allende Prieto, C., et al. 2015, ApJS, 219, 12 Asboth, V., Conley, A., Sayers, J., et al. 2016, MNRAS, 462, 1989 Barger, A. J., Cowie, L. L., Sanders, D. B., et al. 1998, Natur, 394, 248 Becker, G. D., Rauch, M., \& Sargent, W. L. W. 2009, ApJ, 698, 1010 Behroozi, P. S., Conroy, C., \& Wechsler, R. H. 2010, ApJ, 717, 379 Bian, F., Fan, X., Bechtold, J., et al. 2010, ApJ, 725, 1877 Blain, A. W., Chapman, S. C., Smail, I., \& Ivison, R. 2004, ApJ, 611, 725 Bothwell, M. S., Smail, I., Chapman, S. C., et al. 2013, MNRAS, 429, 3047 Bouché, N., Dekel, A., Genzel, R., et al. 2010, ApJ, 718, 1001

Brammer, G. B., Whitaker, K. E., van Dokkum, P. G., et al. 2009, ApJL, 706, L173

Bullock, J. S., Kolatt, T. S., Sigad, Y., et al. 2001, MNRAS, 321, 559

Cameron, E. 2011, PASA, 28, 128

Casey, C. M., Berta, S., Béthermin, M., et al. 2012, ApJ, 761, 140

Casey, C. M., Chapman, S. C., Smail, I., et al. 2011, MNRAS, 411, 2739

Casey, C. M., Narayanan, D., \& Cooray, A. 2014, PhR, 541, 45

Chapman, S. C., Blain, A. W., Smail, I., \& Ivison, R. J. 2005, ApJ, 622, 772

Condon, J. J. 1992, ARA\&A, 30, 575

Coppin, K., Chapin, E. L., Mortier, A. M. J., et al. 2006, MNRAS, 372, 1621

Cowley, W. I., Lacey, C. G., Baugh, C. M., \& Cole, S. 2016, MNRAS, in press (arXiv:1608.04736)

Croom, S. M., Smith, R. J., Boyle, B. J., et al. 2004, MNRAS, 349, 1397

Cushing, M. C., Vacca, W. D., \& Rayner, J. T. 2004, PASP, 116, 362

Dekel, A., \& Birnboim, Y. 2006, MNRAS, 368, 2

Di Matteo, T., Springel, V., \& Hernquist, L. 2005, Natur, 433, 604

Eales, S., Lilly, S., Gear, W., et al. 1999, ApJ, 515, 518

Eales, S. A., Raymond, G., Roseboom, I. G., et al. 2010, A\&A, 518, L23

Elias, J. H., Joyce, R. R., Liang, M., et al. 2006, Proc. SPIE, 6269, 62694C

Fabian, A. C. 2012, ARA\&A, 50, 455

Faucher-Giguere, C.-A., Feldmann, R., Quataert, E., et al. 2016, MNRAS, 461, 32

Faucher-Giguère, C.-A., Hopkins, P. F., Kereš, D., et al. 2015, MNRAS, 449, 987

Fu, H., Cooray, A., Feruglio, C., et al. 2013, Natur, 498, 338

Fu, H., Jullo, E., Cooray, A., et al. 2012, ApJ, 753, 134

Fumagalli, M., Hennawi, J. F., Prochaska, J. X., et al. 2014, ApJ, 780, 74

Greve, T. R., Bertoldi, F., Smail, I., et al. 2005, MNRAS, 359, 1165

Griffin, M. J., Abergel, A., Abreu, A., et al. 2010, A\&A, 518, L3

Hainline, L. J., Blain, A. W., Smail, I., et al. 2011, ApJ, 740, 96

Harris, A. I., Baker, A. J., Frayer, D. T., et al. 2012, ApJ, 752, 152

Heckman, T. M., \& Best, P. N. 2014, ARA\&A, 52, 589

Helou, G., Soifer, B. T., \& Rowan-Robinson, M. 1985, ApJL, 298, L7

Hennawi, J. F., \& Prochaska, J. X. 2007, ApJ, 655, 735

Hennawi, J. F., \& Prochaska, J. X. 2013, ApJ, 766, 58

Hennawi, J. F., Prochaska, J. X., Burles, S., et al. 2006a, ApJ, 651, 61

Hennawi, J. F., Strauss, M. A., Oguri, M., et al. 2006b, AJ, 131, 1

Hickox, R. C., Wardlow, J. L., Smail, I., et al. 2012, MNRAS, 421, 284

Hopkins, A. M., Afonso, J., Chan, B., et al. 2003, AJ, 125, 465

Horne, K. 1986, PASP, 98, 609

Hu, E. M., Kim, T.-S., Cowie, L. L., Songaila, A., \& Rauch, M. 1995, AJ, 110,1526
Hughes, D. H., Serjeant, S., Dunlop, J., et al. 1998, Natur, 394, 241 Hwang, H. S., Elbaz, D., Magdis, G., et al. 2010, MNRAS, 409, 75 Ilbert, O., McCracken, H. J., Le Fèvre, O., et al. 2013, A\&A, 556, 55 Ivezić, Ž., Menou, K., Knapp, G. R., et al. 2002, AJ, 124, 2364 Ivison, R. J., Greve, T. R., Dunlop, J. S., et al. 2007, MNRAS, 380, 199 Ivison, R. J., Magnelli, B., Ibar, E., et al. 2010, A\&A, 518, L31 Ivison, R. J., Papadopoulos, P. P., Smail, I., et al. 2011, MNRAS, 412, 1913 Kelson, D. D. 2003, PASP, 115, 688

Keres, D., Katz, N., Weinberg, D. H., \& Davé, R. 2005, MNRAS, 363, 2 Kochanek, C. S., Eisenstein, D. J., Cool, R. J., et al. 2012, ApJS, 200, 8 Kovács, A., Chapman, S. C., Dowell, C. D., et al. 2006, ApJ, 650, 592 Kroupa, P. 2002, Sci, 295, 82

Magnelli, B., Lutz, D., Santini, P., et al. 2012, A\&A, 539, A155

McMullin, J. P., Waters, B., Schiebel, D., Young, W., \& Golap, K. 2007, in ASP Conf. Ser. 376, Astronomical Data Analysis Software and Systems XVI, ed. R. A. Shaw, F. Hill, \& D. J. Bell (San Francisco, CA: ASP), 127

Michałowski, M. J., Dunlop, J. S., Cirasuolo, M., et al. 2012, A\&A, 541, 85 Michalowski, M. J., Watson, D., \& Hjorth, J. 2010, ApJ, 712, 942

Mo, H., van den Bosch, F. C., \& White, S. 2010, Galaxy Formation and Evolution (Cambridge: Cambridge Univ. Press)

Murphy, E. J., Condon, J. J., Schinnerer, E., et al. 2011, ApJ, 737, 67

Narayanan, D., Turk, M., Feldmann, R., et al. 2015, Natur, 525, 496

Navarro, J. F., Frenk, C. S., \& White, S. D. M. 1996, ApJ, 462, 563

Nayyeri, H., Keele, M., Cooray, A., et al. 2016, ApJ, 823, 17

Neistein, E., \& Dekel, A. 2008, MNRAS, 383, 615

Nguyen, H. T., Schulz, B., Levenson, L., et al. 2010, A\&A, 518, L5

Oke, J. B., Cohen, J. G., Carr, M., et al. 1995, PASP, 107, 375

Oliver, S. J., Bock, J., Altieri, B., et al. 2012, MNRAS, 424, 1614

Papovich, C., Cool, R., Eisenstein, D., et al. 2006, AJ, 132, 231

Perley, R. A., \& Butler, B. J. 2013, ApJS, 204, 19

Pilbratt, G. L., Riedinger, J. R., Passvogel, T., et al. 2010, A\&A, 518, L1

Prochaska, J. X., Hennawi, J. F., Lee, K.-G., et al. 2013a, ApJ, 776, 136

Prochaska, J. X., Hennawi, J. F., \& Simcoe, R. A. 2013b, ApJL, 762, L19

Prochaska, J. X., O’Meara, J. M., Fumagalli, M., Bernstein, R. A., \& Burles, S. M. 2015, ApJS, 221, 2

Rahmati, A., Schaye, J., Bower, R. G., et al. 2015, MNRAS, 452, 2034

Rigby, E. E., Maddox, S. J., Dunne, L., et al. 2011, MNRAS, 415, 2336

Roseboom, I. G., Oliver, S. J., Kunz, M., et al. 2010, MNRAS, 409, 48

Rudie, G. C., Steidel, C. C., Trainor, R. F., et al. 2012, ApJ, 750, 67

Scott, S. E., Dunlop, J. S., \& Serjeant, S. 2006, MNRAS, 370, 1057

Seifert, W., Appenzeller, I., Baumeister, H., et al. 2003, Proc. SPIE, 4841, 962

Silk, J., \& Rees, M. J. 1998, A\&A, 331, L1

Smail, I., Ivison, R. J., \& Blain, A. W. 1997, ApJL, 490, L5

Smith, D. J. B., Dunne, L., Maddox, S. J., et al. 2011, MNRAS, 416, 857

Smolčić, V., Aravena, M., Navarrete, F., et al. 2012, A\&A, 548, A4

Swinbank, A. M., Smail, I., Chapman, S. C., et al. 2004, ApJ, 617, 64

Swinbank, A. M., Smail, I., Longmore, S., et al. 2010, Natur, 464, 733

Tacconi, L. J., Genzel, R., Smail, I., et al. 2008, ApJ, 680, 246

Targett, T. A., Dunlop, J. S., Cirasuolo, M., et al. 2012, arXiv:1208.3464

Toft, S., Smolčić, V., Magnelli, B., et al. 2014, ApJ, 782, 68

Vacca, W. D., Cushing, M. C., \& Rayner, J. T. 2003, PASP, 115, 389

Valiante, E., Smith, M. W. L., Eales, S., et al. 2016, MNRAS, 462, 3146

Viero, M. P., Asboth, V., Roseboom, I. G., et al. 2014, ApJS, 210, 22

Wang, L., Viero, M., Clarke, C., et al. 2014, MNRAS, 444, 2870

Wardlow, J. L., Smail, I., Coppin, K. E. K., et al. 2011, MNRAS, 415 1479

Weiß, A., De Breuck, C., Marrone, D. P., et al. 2013, ApJ, 767, 88

Weiß, A., Kovács, A., Coppin, K., et al. 2009, ApJ, 707, 1201

White, M., Myers, A. D., Ross, N. P., et al. 2012, MNRAS, 424, 933

Yun, M. S., Scott, K. S., Guo, Y., et al. 2012, MNRAS, 420, 957 